\title{
Analog, Digital And Short Pulse Modulation Of Ultrafast Gallium Aluminum Arsenide Semiconductor Lasers
}

Lau, K., Yariv, A.

K. Y. Lau, A. Yariv, "Analog, Digital And Short Pulse Modulation Of Ultrafast Gallium Aluminum Arsenide Semiconductor Lasers," Proc. SPIE 0533, Ultrashort Pulse Spectroscopy and Applications, (2 April 1985); doi: $10.1117 / 12.946547$ 
Analog, digital and short pulse modulation of ultrafast gallium aluminum arsenide semiconductor lasers

$$
\text { K.Y. Lau }
$$

Ortel Corporation

2015 W. Chestnut St., Alhambra, California 91803

$$
\text { A. Yariv }
$$

California Institute of Technology, Pasadena, California 91125

\section{Introduction}

Semiconductor lasers are potentially devices of great importance for optical transmission as well as short pulse generation for various sampling, characteration and dispersion measurements. Since semiconductor lasers are currently driven devices, it is relatively easy to modulate the optical output and to generate short pulses, on the order of $10 \mathrm{ps}$ long, by directly modulating the injection current into the laser. This paper will present some recent developments in injection lasers which are capable of being analog or digitally modulated at rates up to $10 \mathrm{GHz}$, as well as generating short optical pulses at repetition rates from several hundred megahertz to tens of gigahertz.

\section{Approach to high speed lasers}

A basic analysis shows that the small signal modulation bandwidth is

$$
f_{r}=\frac{1}{2} \frac{\pi}{\sqrt{\mathrm{AP}_{\mathrm{p}}}}
$$

where $T p$ is the photon lifetime, given by $\mathrm{T}_{\mathrm{p}}=1 / \mathrm{v}(\alpha+1 / \mathrm{L} \ln 1 / \mathrm{R})$ where $\mathrm{v}$ is the group velocity of the light, $\alpha$ is the distributed loss, $L$ is the length of the cavity and $R$ is the mirror reflectivity, $\mathrm{P}_{0}$ is the steady state photon density and $A$ is the differential optical gain constant. The modulation bandwidth of the laser is widely accepted to be equal to fr. A means to increase the modulation speed of semiconductor lasers is to decrease the cavity length and hence the photon lifetime. The actural device used was a buried heterostructure laser fabricated on a semi-insulating substrate (BH on SI) [3]. In addition to a low lasing threshold (typically $\leq 15 \mathrm{~mA}$ ) which is necessary to avoid excessive heating due to the short cavity length, these lasers possess very low parasitic capacitance [4] which is detrimental at high speeds. The modulation bandwidth can be pushed to beyond $8 \mathrm{GHz}$ by operating near the catastrophic damage point. A second method to increase the modulation bandwidth is to increase the optical gain constant by lowering the operating temperation of the laser. The relative slopes of the plots of bandwidth versus the square root of the emitted optical power () yield values for the relative change in $A$ as the temperature is varied. The ratio of the slope at $22^{\circ} \mathrm{C}$ to that at $-50^{\circ} \mathrm{C}$ is 1.34 . This factor is fairly consistent (between 1.3 and 1.4) among all the lasers tested, even including those from different wafers. This infers that the intrinsic differential optical gain increases by a factor of $\cong 1.8$ by cooling from $22^{\circ} \mathrm{C}$ to $-50^{\circ} \mathrm{C}$, which is consistent with theoretical gain calculations [5]. A third parameter to be tackled is the optical power density $\mathrm{P}_{0}$. However, an increase of the optical power can bring about undue degradation or even catastrophic damage to the laser unless the structure of the laser is suitably designed. One common means of raising the ceiling of the reliable operating power of semiconductor lasers is by means of a large optical cavity [6]. The mechanism responsible for a higher catastrophic damage power in these devices is by lowering the optical power density at the active layer, since such damage commonly originates from the active layer near the crystal facet. This maneuver, however, serves little to increase the modulation bandwidth because the quantity of concern here, the photon density within the active region ( $P_{0}$ in Eq. (7)), remains unchanged. A laser suitable for high speed operation should therefore be one with a tight optical confinement in the active region along the entire length of the laser, with a transparent window at the end regions able to withstand much larger photon densities without catastrophic damage. The use of a transparent window structure to increase the catastrophic damage level has previously been demonstrated $[7,8]$. Using a window buried heterostructure laser fabricated on a semi-insulating substrate, we have demonstrated for the first time a direct modulation bandwidth exceeding $10 \mathrm{GHz}$ for a semiconductor laser operating at room temperature. The device used is structurally similar to the BH on SI laser except that here the end regions near the facets is covered by a layer of unpumped GaAlAs which forms a transparent window. The optical wave propagates freely in the end window region. As a result of diffraction, only a small amount of light reflected from the crystal facet couples back into the active region. The theoretical value of the effective reflectivity, assuming a fundamental gaussian beam profile, is $\cong 5 \%$ for $L=5 \mu \mathrm{m}$. The actual values of $L$ for the devices fabricated lie around this value. The 
threshold current of window BJ on SI lasers ranges from $14 \mathrm{~mA}$ to $25 \mathrm{~mA}$. age threshold in these devices is beyond $120 \mathrm{~mW}$ under pulse operation. the maximum operating power is limited by heating to $50 \mathrm{~mW}$. The - 3dB bandwidth can be pushed to beyond $10 \mathrm{GHz}$ when operating above $15 \mathrm{~mW}$. The bandwidth characteristics of a large optical cavity $\mathrm{BH}$ on SI lasers is also investigated, and found to be substantially lower at comparable output power levels. This bears witness to the fact that the optical power density at the active region of these devices is lower than that in lasers of conventional construct ion.

\section{Short pulse generation}

There are various means of generating short pulses from a semiconductor laser. The easiest way is to directly modulate the laser diode with short, intense current pulses or by a large-amplitude sinusoidal signal. Optical pulses on the order of 20 ps can be generated in this manner. If shorter pulses are desired, the method of mode-locking can be used. The laser is coupled to an external optical cavity, and by means of active (directly modulating the laser at the round trip frequency of the external cavity) or passive (by some saturable absorning defects in the laser) optical pulses below $10 \mathrm{ps}$ can be generated at the round trip frequency of the external cavity. The need for very sensitive alignment in coupling the laser to the external cavity is a drawback in practical application of mode-locked laser systems. One way of generating short pulses of below 10ps without the need of external pulse of RF pumping, or sensitive optical alignment is to apply optoelectronic feedback to a self-pulsing semiconductor laser. Highly stable optical pulses (with a frequency jitter of $<30 \mathrm{kHz}$ ) of pulsewidths between 10 to 20 ps can be generated, with a very high repetition rate from 1-5 GHz which can be electrically tuned.

The lasers used in this experiment were similar to the high speed GaAlAs lasers described above, which possesses the highest direct modulation speed reported thus far. Selfpulsation was induced in these otherwisw well-behaved lasers by momentarily operating the lasers beyond the catastrophic damage level. It must be emphasized here that it is not necessary for one to induce damage to a laser for it to self-pulse; a highly reliable way of accomplishing the task is by a double contact laser structure reported previously. This frequency ( $f$ ) of pulsation varies with the pump current (i) following a dependence as in conventional relaxation oscillation in lasers indicated in the last section.

Next, the output of the laser is coupled to a high speed GaAs pin photodiode, the output of which is amplified by a $2-4 \mathrm{GHz}, 20 \mathrm{~dB}$ gain amplifier, which in turn drives the injection laser. The intensity output from the laser now pulsates, with a much reduced linewidth, at an integral multiple of $f_{0}$ which is closest to the "natural" self-pulsation frequency of the solitary laser (without optoelectronic feedback), where $f_{0}$ is the inverse of the total

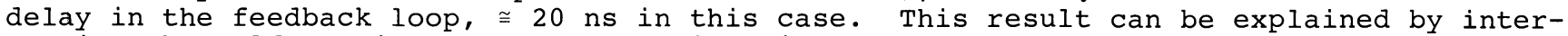
preting the self-pulsing laser as a device with a very high gain in a narrow frequency band around the self-pulsing frequency, and hence oscillation commences at the integral multiple of $f_{0}$ which has the highest open-loop gain. As the natural self-pulsing frequency is tuned by varying the pump current, the pulsation frequency jumps from one integral multiple of $f_{0}$ to the next. The pulsation repetition rate can be steptuned from $1.2 \mathrm{GHz}$ to $4.5 \mathrm{GHz}$ by varying the pump current.

The exact optical pulsewidth was measured by a standard optical doubling autocorrelation technique using $\mathrm{LiIO}_{3}$ as the non-linear medium. During these measurements, the loop gain is fixed at a value of 7. The pulsewidth varies between 20 to 12 ps within the repetition-rate tuning range. In contrast, when the optoelectronic loop is opened, the pulse width of the self-pulsing laser is $\cong 57 \mathrm{ps}$.

Next, the wideband microwave amplifier $(1-6 \mathrm{GHz}, 20 \mathrm{~dB}$ gain) is replaced by a high gain (39.5 dB) narrow band amplifier $(1.8-2 \mathrm{GHz})$ which produces a loop gain of 65 . Under this increased loop gain, the measured pulsewidth falls below $10 \mathrm{ps,}$, the shortest being $9.5 \mathrm{ps}$ at a pulse repetition rate of $\cong 2 \mathrm{GHz}$.

\section{Digital modulation}

The results described in Section 2 of this paper are concerned with small signal modulation. By "small signal" one means that the modulation depth in the optical output of the laser is substantially less than one. In wideband digital telecommunication, the on-off ratio in the laser output should be as large as possible to avoid excessive power penalty. The modulation response of a laser under large modulation is different from (and generally worse than) the small signal response, although the latter is often a good indicator of the former as long as the laser is maintained above threshold at all times. Ikegami and Suematsu [9] studied large signal effects by direct numerical integration of the rate equations. The result is quite similar to the small signal response except that the relaxation oscillation occurs at a lower frequency. The reason for this is that the laser cannot be swiftly brought from a state of very low optical level to a high level due to the low rate of stim- 
ulated emission at the low-power state. This can be illustrated by modulating a laser, which is biased at a high optical level, with two closely spaced negative-going current pulses. When the optical modulation depth is below 70-80\%, optical output is a faithful reproduction of the current pulse input up to a pulse spacing of $200 \mathrm{ps}$, which is limited by the current pulse width. When the optical modulation depth exceeds $\cong 85 \%$, the laser responds sluggishly when going from the "low" to the "high" state, and severe relaxation oscillation occurs. This is obviously not satisfactory for a pulse-code modulation transmission. The slow rate of the optical output in the large signal regime has been calculated from the rate equations by Kaminow and Tucker [10], and the relaxation oscillation associated with large modualtion depth has recently been observed by Eisenstein et al. [11]. The basic conclusion of this section is that when high speed modulation is desired, the optical modulation depth should be maintained below $\cong 70 \%$, but when power penalty must be minimized in a digital telecommunication system, modulation speed will be sacrificed.

\section{References}

1. H.W. Yen and M. Barnoski, Appl. Phys. Lett., 32, 182, 1978 . 59,1980 .

2. G. Lutes, Jet Propulsion Lab, Telecommun. and Data Acquisition Progress Report, 42,

3. N. Bar-Chaim, J. Katz, I. Ury, A. Yariv, "Buried heterostructure AlGaAs laser on semi-insulating substrate", Electron. Lett., 17, pp. 108-109, 1981.

4. I. Ury, K.Y. Lau, N. Bar-Chaim, and A. Yariv, "Very high frequency GaAlAs laserfield effect transistor monolithic integrated circuit", Appl. Phys. Lett., 41, pp. 126-128, 1982 . 1978 .

5. H.C. Casey and M.B. Panish, Heterostructure Lasers, part A, pg. 174, Academic Press

6. H. Kressel and J.K. Butler, Semiconductor Lasers and Heterojunction LED's, Academic Press, New York, 1975, pp. 230.

7. S. Takahashi, T. Kobayashi, H. Saito and Y. Furukawa, "GaAs-AlGaAs DH lasers with buried facets", Jap. J. Appl. Phys., 17, pp. 865-870, 1978.

8. H. Blauvelt, S. Margalit and A. Yariv, "Large optical cavity AlGaAs buried heterostructure window laser", Appl. Phys. Lett., 40, pp. 1029-1031, 1982 .

9. I.P. Kaminow, "High speed modulation of semiconductor lasers", Conf. on Optical Fiber Communication, paper NJ1, New Orleans, 1984.

10. R.S. Tucker and I.P. Kaminow, "high speed switching transients in directly modulated InGaAsP lasers", CLEO '84, paper FF1, Anaheim, 1984 .

11. G. Eisenstein, U. Koren, R.S. Tucker, B.L. Kasper, A.H. Gnauck and P.K. Tien, "High speed analog and digital modulation of $1.51 \mu \mathrm{m}$ wavelength three channel buried crescent InGaAsP lasers", Appl. Phys. Lett., 45, pp. 311-313, 1984. 